\title{
Incidencia de las variables sociodemográficas en los patrones de consumo del tabaco en estudiantes universitarios ${ }^{1}$
}

\author{
The incidence of sociodemographic variables in the tobacco \\ consumption patterns of university students
}

\author{
Ana Sandra Aguilar de Mendoza² \\ Investigadora Universidad Tecnológica de El Salvador \\ ana.aguilar@utec.edu.sv \\ Recibido: 17/01/2015 - Aceptado: 25/03/2015
}

\section{Resumen}

Con el objetivo de analizar el patrón de consumo de tabaco en estudiantes universitarios, se pretendió identificar las variables sociodemográficas que inciden en el fortalecimiento del patrón de consumo de tabaco y los comportamientos relacionados con la compra, así como los lugares donde consumen el tabaco, y el conocimiento de los daños que genera la nicotina a través de la propaganda en los medios de comunicación. Se realizó un estudio descriptivo, exploratorio, con un diseño ex post facto en una muestra de 377 estudiantes seleccionados aleatoriamente, representativa del $95 \%$ y con un margen de error del 0,5\%. Los resultados evidencian que el grupo que está más expuesto al consumo de tabaco está en un rango de edad entre los 19 a los 20 años. La edad de inicio de consumo de tabaco entre los participantes fue en promedio a los 15 años; la prevalencia de vida entre los fumadores es del $46,15 \%$. El estado económico y

\section{Abstract}

With the objective of analyzing the tobacco consumption pattern of university students, a study was conducted in order to identify the sociodemographic variables that influence the strengthening of such pattern and the behaviors related to the purchase, place of consumption, and awareness of the damages generated by nicotine as advertised in the media.

This was a descriptive, exploratory study with an ex post facto design in a sample of 377 students who were randomly selected and who represented 95\% [of the population]; the margin of error was $0,5 \%$.

The results showed evidence that those who are mostly exposed to tobacco consumption are between 19 and 20 years of age. The average age in which individuals started using tobacco was 15; the lifetime prevalence of smokers is $46,15 \%$. The financial and work status have no influence in tobacco consumption.

La presente investigación es el resultado de un proyecto conjunto con otras universidades y la Universidad Tecnológica de El Salvador. Las solicitudes de información, separatas y otros documentos relativos al presente estudio pueden hacerse a la dirección postal: Universidad Tecnológica de El Salvador, Vicerrectoría de Investigación, Dirección de Investigaciones, Calle Arce y 17a. Avenida Norte, edificio José Martí, No. 1020, o al correo electrónico: ana.aguilar@utec.edu.sv

Licenciada en Psicología. Investigadora y docente de la Utec. correo electrónico ana.aguilar@utec.edu.sv 
el laboral no influyen en el consumo. El estado civil soltero sí incide en fumar. En el último mes, el consumo de cigarrillos declarado fue entre 1 a 3 diarios. Los que consumen más cigarrillos diarios presentan mas años de estarlos consumiendo, demostrando una dependencia de la nicotina. La presión grupal es la que más incide en este grupo para que fume. En el recinto universitario es donde fuman con mayor frecuencia; y las actividades preventivas, a través de los medios de comunicación, no logran permear a los estudiantes universitarios.

\section{Palabras clave}

Tabaquismo, prevalencia de consumo, dependencia de la nicotina.
Those who were listed as single showed an incidence in smoking. [When asked about their consumption] during the past month, they reported smoking 1-3 cigarettes daily. Also, those who consumed more cigarettes per day were the same ones with the most years of smoking, thus demonstrating nicotine dependence.

Peer pressure incided most in this group. They smoke more frequently at the university premises; prevention activities through the mass media do not influence university students.

\section{Keywords}

Smoking, consumption prevalence, nicotine dependence.

\section{Introducción}

Se ha abordado ampliamente el tema del consumo del tabaco en varios países y en diferentes contextos. Sin embargo, perfilar el fenómeno se vuelve cada día más complejo debido a que cada año hay nuevos consumidores de los que, de continuar en el futuro consumiendo tabaco como lo vienen haciendo, tendremos a corto plazo un incremento en sus necesidades de salud, por la presencia de enfermedades crónicas debido a la adicción al tabaco (Kuri-Morales, Hoy y Cortéz-Ramírez, 2006).

El tabaco es la sustancia psicoactiva que consumen las personas, y su edad de inicio en estudiantes universitarios chilenos se situó en los 13.1 años (Antona, 2008). En Venezuela, en un estudio realizado en el 2007 en una muestra de 1055 estudiantes universitarios, se encontró que el 17,3 \% consumía tabaco inhalado, y estos habían iniciado su consumo antes de los 20 años de edad (Gómez, Herde, Lafee, Lobo y Martín, 2007).

En estudios a nivel universitario, en Chile, se encontró un porcentaje de consumo de tabaco del $27 \%$ en estudiantes universitarios (Antona, 2008). En otro estudio en estudiantes universitarios en Barcelona, la prevalencia de fumadores actuales es del $40,8 \%$ de los participantes de una muestra de 400 estudiantes (Prat-Marin, 1994).
En estudios realizados a escalas local y mundial, las variables sociodemográficas son importantes de tipificar en cuanto a que, a partir de ciertos hallazgos, se puede abordar más temprano la prevención de enfermedades psicosociales como las producidas por el consumo de tabaco. Está demostrada ampliamente la relación entre enfermedades cardiovasculares, respiratorias, cáncer y otras con el consumo de tabaco (Antona, 2008).

En el patrón de consumo de tabaco la edad es una variable sociodemográfica significativa para poder estimar a través de los años quiénes, según su género, están consumiendo el tabaco. En Chile se realizó, en el 2010, un estudio observacional en 2.045 estudiantes universitarios; y los resultados fueron que la edad promedio de consumo fue de 20.4 años, con una $\mathrm{dt}=1.6$ años (Zuzulich, Cabieses, $\mathrm{y}$ Pedrals, 2010).

En una muestra de estudiantes españoles de secundaria, en el curso académico 97-98, el 58,1\% de los estudiantes consumía tabaco alguna vez en la vida, en contraste con el 64,1 \% de consumo en las estudiantes (Sáiz et al., 1999). Saiz, en sus resultados, concluye que hay un mayor consumo de tabaco en el sexo femenino, el cual es superior al de los hombres. En estudios de Prat-Marín (1994) no se encontraban diferencias estadísticas significativas según 
el sexo. Esto implica que, a medida que han pasado los años, el consumo de tabaco en la población femenina ha ido sufriendo un proceso de feminización que perfila que el consumo de tabaco es similar en hombres y mujeres (Calleja, Pick, Reidl, y González-Forteza, 2010) .

En estudios sobre estudiantes chilenos, se encontró que el nivel socioeconómico está asociado positivamente al consumo de tabaco durante el último año (p 0.05), cuya muestra presentó, en un $70,3 \%$, un nivel socioeconómico alto (Zuzulich et al., 2010).

En estudios de Bautista (2005), en la muestra utilizada para el estudio de drogas, el $68,9 \%$ de los participantes eran estudiantes a tiempo completo, solteros(as); el 17,5\% trabaja parcialmente y el $24,6 \%$ a tiempo completo.

En El Salvador se encontraron diferencias estadísticas significativas en la prevalencia del consumo de tabaco en el último año en estudiantes universitarios, con respecto al género (Bautista, 2005). Para Antona (2008), en Europa los hombres fuman más que las mujeres. El consumo simultáneo de cigarrillo más marihuana fue baja $(0,2 \%$ y de cigarrillo más drogas no se encontró consumo en la muestra (Bautista, 2005).

En los estudios de Antona (2008), el consumo por día de cigarrillos evidenció que fumadores (hombres y mujeres) de 16 años o más se fuman alrededor de 15 cigarrillos diarios, y el $17 \%$ de los fumadores consumen 20 o más cigarrillos.

En los estudios de Gómez et al. (2007), el 63,7 \% de los universitarios consume menos de media cajetilla; el 15,4 $\%$ consume una cajetilla diaria; y un 19,1 \% más de una cajetilla diaria.

Los entornos familiares y sociales son los principales reforzadores para desarrollar un comportamiento fumador. En los casos en que los padres fuman, estos comportamientos son considerados normales para los jóvenes y con facilidad pueden imitar esta actitud hacia fumar (Sánchez-Zamorano, n.d.)

El consumo de tabaco guarda relación con el conocimiento y las convicciones respecto a la salud; las opiniones negativas serán sostenidas por los no fumadores, mientras que los fumadores presentan menos conocimiento, no personalizan los riesgos y no ven a corto plazo los daños, dada las ventajas de nuevas amistades o reforzamientos grupales que compensan las amenazas para la salud (Barrueco, Hernández, y Torrecilla, 2003).

En los estudios por Bautista (2005), los estudiantes consiguen las sustancias psicoactivas, en el $12 \%$, fuera de la universidad; y dentro de la universidad se presentan porcentajes bajos $(0,8 \%)$, enfatizando que, aunque presente una frecuencia de consumo bajo, en el recinto universitario es necesario el control de esta demanda. Así mismo, el consumo de sustancias psicoactivas, incluyendo el tabaco, es del $2,4 \%$.

En Europa, la presentación del tabaco aceptada para su consumo es la del cigarrillo empaquetado, (27,0 \%), más que el tabaco de liar (5\%) o el puro ( $1 \%$ ). El consumo de cigarrillo está presente en $31 \%$ de los hombres y $23 \%$ de las mujeres (Antona, 2008).

La publicidad comercial del tabaco es concebida para aumentar su consumo, obteniendo nuevos consumidores y alterando el papel del tabaco en la sociedad. El pago de las empresas por campañas publicitarias costosas indica que obtienen grandes ganancias por el consumo del tabaco procesado (Poza, Robledo, Fresnillo, Fresnillo y Fernández, s.f.)

\section{Método}

Es un diseño descriptivo, ex post facto, realizado en la Universidad Tecnológica de El Salvador, como parte de un proyecto multicéntrico de varias universidades.

La población universitaria es de 19.365 estudiantes, para el año 2013. La muestra representa una selección aleatoria de 377 participantes, con un nivel de confianza del $95 \%$ y un margen de error del $5 \%$, calculados para poblaciones finitas, utilizando el programa electrónico Survey Sistem Calculator.

La muestra presentó las siguientes características: el 41,9\% de los estudiantes está inscrito en la Facultad de Ciencias Sociales; el $24,1 \%$ en la Facultad de Ciencias Aplicadas; 
el 22,5\% en la Facultad de Ciencias Empresariales; el $11,4 \%$ en la Facultad de Ciencias Jurídicas; distribuida según carreras, así: el 19,6 \% pertenece a la Licenciatura en Comunicaciones; el 13,5 \% está matriculados en la Licenciatura en Administración de Empresas; el 12,2 \% estudia Licenciatura en Idioma Inglés; el 11,7 \% en Ingeniería en Sistemas y Computación; el 11,4 \% en Licenciatura en Ciencias Jurídicas, y en porcentajes menores al 10,0 \% pertenecen a las licenciaturas en Psicología, Mercadeo, Contaduría Pública, Administración Turística, Diseño Gráfico, Negocios Internacionales, Antropología, Arqueología; Ingeniería Industrial, y a los técnicos en Idioma Inglés, en Periodismo y en Diseño Gráfico.

En la encuesta participaron el 47,2 \% del sexo masculino y el $52,8 \%$ del femenino. Según rangos de edad: entre los 16 a 18 años, se encontró el 11,7 \% de la muestra; entre los 19 a 20 años, el 26,8 \% de los participantes; entre los 21-22 años, el 20,2 \%; entre 23-24 años, el 14,1\%; y de 25 a 47 años, el $26,8 \%$.

Se utilizó un cuestionario autoinformado y adaptado sobre prevalencia del consumo de tabaco, aplicado mediante la técnica de encuesta. El cuestionario incluyó variables sociodemográficas y una escala de consumo de tabaco. Las variables nominales presentes en la escala permitieron un análisis estadístico con pruebas no paramétricas. Los datos recolectados se procesaron en el programa estadístico SSPS 19 y Excel.

\section{Resultados}

En cuanto a las variables sociodemográficas, el 50,1 \% califica su economía como regular; el 35,3 \% como buena; el 6,1 \% como muy buena; y el 5,6 \% como mala, con porcentajes menores al $2 \%$ como muy mala. En cuanto a su estado civil, el 81,4 \% está soltero(a), el 1,7 \% casado, el 2,9\% en unión consensual; y con porcentajes menores al $1,0 \%$ divorciados y viudos. El 15,6 \% declaró tener hijos y el $84,4 \%$ no tenerlos. En cuanto a la variable laboral, el $57,3 \%$ solo estudia y el $42,7 \%$ estudia y trabaja. De este último grupo, el $5.3 \%$ trabaja las 44 horas laborales establecidas a la semana. Al 70,6 \% de los estudiantes son los padres los que le pagan los gastos de habitación, y un $23,9 \%$ trabaja para sostenerse; a menos del 5,0 \% le paga otras personas. En cuanto a los gastos de alimentación, al $66,6 \%$ se lo pagan sus padres; el $27,1 \%$ paga sus alimentos de su salario.

Al preguntar sobre el pago de su colegiatura, al 56,5\% le es pagada por sus padres; el 36,1\% por el trabajo que desempeñan; al 8,0 \% se la pagan otras personas, y menos del 2 \% goza de una beca. Los libros en un 56,5 \% son pagados por sus padres, y un $36,1 \%$ por su salario menos del $10,0 \%$ es pagado por otras personas. Para transportarse, a un $56,0 \%$ se lo pagan sus padres, y un $36,1 \%$ lo paga de su trabajo. Al 57,8 \% le es difícil asumir los costos de sus estudios, al 18,6\% les es fácil asumir los costos para estudiar, y a un 18,0 \% le es muy difícil. A menos del 6,0 \% le es muy fácil.

En cuanto al consumo de tabaco, el 53,5 \% declaró no fumar y el $46,5 \%$ sí fuma. La esperanza de vida es del 46,15\%. Los estudiantes iniciaron el consumo de tabaco a los 15 años, como promedio, pero declararon casos en que su inicio fue a los 9 años. La edad mayor se situó a los 24 años.

La incidencia de los casos se registró, en el 41,9\%, hace más de un año; el 54,4 \% no contestó, y el 2,7 \% reportó un consumo hace más de un mes, pero menos de un año; el 1,1 \% manifestó que su último consumo fue entre los últimos 30 días.

Al establecer la dependencia del cigarro, el 88,9\% no ha fumado al menos 100 cigarrillos en la vida, pero el 27,6\% ha fumado menos de esta cantidad y $61,3 \%$ no contestó; el $11,1 \%$ sí lo ha fumado. Sobre la prevalencia año, el 21,0 \% ha fumado tabaco en los últimos doce meses, y 77,7 \% declaró no haberlo hecho. En cuanto a la prevalencia mes, el 16,2 \% declaró haber fumado tabaco durante los últimos 30 días. El 4,8 \% ( $n=18)$ fumó todos los días del mes. El 1,3 \% fumó durante 20 días en el mes, 21,0 \% fumó entre 10 a 15 días por mes; 2,7 \% fumó entre 5-9 días en el mes; y 6,2 \% fumó entre uno a cuatro días en el mes.

Los cigarrillos consumidos por día en el último mes oscilan entre 1 a 60 diarios. El 6,1 \% consume de uno a dos cigarrillos, entre 3 a 5, entre 8 a 10, más de una cajetilla, más de dos cajetillas. Hay un porcentaje significativo que no contestó. Los estudiantes, en el 79,3 \%, niegan un consumo de tabaco anterior al año; y el 11,7 \% no contestó. 
Sin embargo, el 3,8 \% es de fumadores con de 3 a 5 años de consumo. El 3,7 \% ha fumado en un rango de 6 a 10 años consecutivos; y el 1,7\% es de fumadores con de 10 a 15 años de consumo.

Se exploró el entorno social y familiar, encontrándose que en el $29,2 \%$ de los participantes se encuentra alguien de la familia que consume cigarrillos. Los lugares donde han fumado en los últimos treinta días son, en primer lugar, en la universidad $(12,5 \%, \mathrm{n}=47)$; en segundo, lo han hecho en la casa de los amigos el 6,1 \% de los participantes; en lugares públicos, el 3,4 \%; y en su casa, el 2,9\%.

El lugar donde los estudiantes han comprado en los últimos treinta días los cigarrillos es en la tienda, el $15,4 \%$ de los participantes; en el supermercado, el 3,7 \%; y en ventas ambulantes, el 2,9\%. La compra en discotecas, bares y otros lugares de uso colectivo no reporta porcentajes mayores o iguales al $1 \%$.
Entre las marcas de cigarrillo que más consumen se evidencia Marlboro, en un consumo del 43,5 \%; y Diplomat, en un del 21,2\%; los cigarrillos Pall Mall son consumidos en un $9,4 \%$ de los participantes. La mezcla en el consumo de varias marcas se presenta en el $15,3 \%$ de los fumadores; y el consumo del resto de marcas de cigarrillos no pasa el $1 \%$ (puro, hierba del mal punto rojo). La cantidad de dinero gastada en cigarrillos por los estudiantes es, en primer lugar, de menos de un dólar en el $44,7 \%$ de los consumidores; de un dólar, en el 36,5\%; el 14,1 \% gasta entre 4 y 5 dólares en cigarrillos; y el 4,7 \% gasta entre 6 a 7 dólares en la compra de cigarros.

En cuanto a las actividades de prevención, promoción y fomento de conductas y estilos de vida saludables, se les preguntó si observaban propaganda favorable al consumo de tabaco. Los resultados fueron los siguientes (ver tablas 1 y 2 ).

Figura 1.

Propaganda escuchada por los estudiantes sobre el consumo de tabaco

\begin{tabular}{|l|c|c|c|c|}
\hline Propaganda escuchada & Mucha \% & $\begin{array}{c}\text { Poca } \\
\%\end{array}$ & $\begin{array}{c}\text { Ninguna } \\
\%\end{array}$ & Total\% \\
\hline A favor del consumo de tabaco & 35,5 & 43 & 21,3 & 100 \\
\hline Antipropaganda a no fumar & 18,6 & 38,2 & 43,2 & 100 \\
\hline
\end{tabular}

Figura 2.

Propaganda percibida en los medios de comunicación por los estudiantes universitarios

\begin{tabular}{|c|c|c|c|c|}
\hline $\begin{array}{c}\text { Propaganda en medios de comunicación } \\
\text { (revistas, periódicos, radio, televisión y en la vía } \\
\text { pública) }\end{array}$ & Mucha \% & $\begin{array}{c}\text { Poca } \\
\%\end{array}$ & $\begin{array}{c}\text { Ninguna } \\
\%\end{array}$ & $\begin{array}{c}\text { Total } \\
\%\end{array}$ \\
\hline A favor del consumo de tabaco & 27,3 & 48,8 & 23,9 & 100 \\
\hline
\end{tabular}

Al preguntárseles si consideraban que fumar es dañino para la salud, el 92,3 \% sí percibe el riesgo de daño; y el 7,7 \% no lo considera como un riesgo para la salud. En cuanto al daño que puede ocasionar el humo del cigarro en la salud de los otros, el 93,4 \% sí estima que es dañino y el 0,8 \% que no lo es; el 5,3\% manifiesta que no sabe y un 0,5 \% no contestó. En cuanto a la creencia que si fumar hace bajar de peso, el
43,5 \% declaró que no creen eso; y el 23,1 \% cree que sí se baja de peso. El $32,4 \%$ no sabe y el $1 \%$ no contestó.

Finalmente, se les preguntó si habían recibido alguna capacitación sobre evitar el consumo de cigarrillos; y los resultados fueron que en el $73,7 \%$ no la ha recibido; el 18,3 \% sí la ha recibido y un 8 \% no contestó. 


\section{Análisis y discusión de resultados}

En el grupo participante de este estudio, el 53,5 \% declaró que no fuma y el $46,5 \%$ que sí. El grupo de edad más expuesto al consumo del tabaco en los estudiantes universitarios es entre los 19 a 20 años (26,8 \%), seguido por el grupo de 25 años a más (26,8\%). Este consumo de tabaco es más alto que el evidenciado por Prat-Marín en el 2004 (PratMarin, 1994), cuya prevalencia fue del $40,8 \%$; y más alto que el encontrado por Antona en el 2008, cuyo porcentaje de consumo de tabaco en estudiantes universitarios fue del $27,0 \%$ (Antona, 2008). Concuerda este estudio con las edades de consumo.

Al igual que en países europeos, el consumo de tabaco más aceptado es el cigarrillo empaquetado (Antona, 2008). La edad de inicio de consumo de tabaco fue a los 15 años, como promedio; pero declararon casos en los que su inicio fue a los 9 años. La edad mayor se situó en los 24 años. Eso concuerda con los estudios de Antona (2008), en donde la edad de inicio de los fumadores fue a los 13 años, en promedio. Y antes de los 20 años, en los estudios de (Gómez et al., 2007), en estudiantes universitarios venezolanos.

La prevalencia vida entre los fumadores es del $46,15 \%$ (n $=174$ ). La prevalencia vida en mujeres es del $35,6 \%$ ( $n=$ 62); y la prevalencia vida en los hombres es del $64,4 \%(n=$ 112). En muestras españolas para años anteriores (1999), se percibía un consumo femenino de tabaco del $64,1 \%$, el cual era superior al de los hombres. Sin embargo, las diferencias estadísticas entre los sexos son significativas $(t=-6.45, p=$ 0.000 ) en la muestra salvadoreña. Podemos concluir que el consumo de tabaco en los estudiantes universitarios no está sufriendo un proceso de feminización, ya que el consumo de tabaco no es similar entre hombres y mujeres.

La economía de los estudiantes es calificada por los participantes como regular (50,1\%); y el 35,3 \% la declaró como buena. Este estado económico presenta diferencias significativas entre hombres y mujeres; los hombres la consideran regular-buena y las mujeres buena-regular. Sin embargo, tanto los que han fumado como los que no declaran el mismo estado económico $\left(X^{2}=.250\right)$. Se puede concluir que el estado económico no influye en el consumo de tabaco en los estudiantes universitarios. En estudios realizados en Chile, el nivel socioeconómico alto estaba asociado positivamente al consumo de tabaco, fenómeno que no ocurre en la población salvadoreña, ya que los consumidores frecuentes se localizan entre estados económicos regulares y buenos, principalmente.

El estado civil más predominante entre los fumadores y no fumadores es ser soltero (81,4 \%), sin embargo, encontramos diferencias significativas entre los fumadores solteros $(37,4 \%, n=140)$ y los otros estados, como casados, de unión consensual, divorciados y viudos. Estos resultados evidencian que es más fácil fumar siendo soltero que en cualquier otro estado civil. Esto concuerda con el estudio de Bautista en la muestra universitaria en el año 2005 (Bautista, 2005).

Con respecto al estado laboral, la muestra de este estudio difiere a los estudios de Bautista (Bautista, 2005), en donde los porcentajes de estudiantes que trabajaban parcialmente o a tiempo completo ( $17,5 \%$ y $24,6 \%$, respectivamente) son menores al $42,7 \%$ de los que estudian y trabajan. No se encontraron diferencias significativas entre los fumadores que estudian y trabajan o solo estudian $\left(X^{2}=6.849, p=.144\right)$. Se concluye que el estado laboral no incide en el consumo de tabaco.

A los participantes sus padres pagan sus costos de vida: habitación, comida, colegiatura, trasporte, libros. Manifiestan su dificultad para asumir los costos de sus estudios $(57,8 \%)$.

Se evidenció una incidencia de consumo de tabaco, en el último mes, del $1.1 \%$; una prevalencia mes del 16,2 \% y una prevalencia año del $21 \%$. El $11,1 \%$ ha fumado al menos 100 cigarrillos en su vida. En esta pregunta, un gran porcentaje de participantes no contestó.

Los estudiantes en diferentes edades fuman entre 1 a 3 cigarrillos diarios. En el grupo de edad de 21 a 22 años fuman entre 4 y 5 cigarrillos. Tres estudiantes en edades de 25 a más años fumaron media cajetilla (10 cigarrillos) al día durante el último mes; y un fumador de entre 21 y 22 años de edad fuma aproximadamente 15 cigarrillos. Un estudiante en el rango de edad entre los 16 a 18 años fuma por lo menos una cajetilla al día (20 cigarrillos). Esta tendencia es diferente al estudio de Antona, en donde los más jóvenes 
fumaban más cigarrillos diarios (16 años: 15 cigarrillos). Sin embargo, evidenciaba en el mismo estudio que el $17 \%$ de los fumadores consumían 20 o más cigarrillos al día (Antona, 2008). En la muestra de universitarios salvadoreños, el $54 \%$ de los que fumaron en el último mes consume entre 1 a 3 cigarrillos diarios; y luego se encuentran casos de consumo desde media cajetilla hasta una cajetilla y media, declarados en distintos grupos de edades. Este patrón de consumo difiere de los encontrados en otros estudios (Antona, 2008; Gómez et al., 2007).

Esta dispersión del consumo diario tiene relación con los años de consumo, aunque un 79,3 \% negó un consumo de tabaco anterior al año; pero los fumadores que consumen más cigarrillos diarios presentan más años de estar inhalando nicotina. Esto demuestra la creación de dependencia de la nicotina a través de los años.

El 29,2 \% de los participantes declaró que tenía un familiar que consumía cigarros, Al analizar a los fumadores se evidenció que solo el 35,4 \% tenía un familiar; y que el 64,6\% restante de fumadores no tenía familiar que le modelara este comportamiento. Se concluye que en esta muestra no son los familiares los principales reforzadores y que posiblemente sean las presiones grupales las que refuercen este estilo de comportamiento. Por lo que quedaría para una próxima investigación esta variable, según (Sánchez-Zamorano, n.d.) los familiares y el entorno social son los principales reforzadores del consumo de tabaco. Esto se correlaciona con las declaraciones de los estudiantes que han consumido tabaco en la universidad durante el último mes $(12,5 \%, n=47)$; y fuera de la universidad, 12,4 \% (casa de amigos, lugares públicos y en su casa de habitación).

En cuanto a los lugares donde compran el cigarro, es principalmente en la tienda (15,4\%); y en las ventas ambulantes, el 2,9\%. Esto es congruente con los estudios de Bautista, que encontró que los estudiantes consumen tabaco en el $2,4 \%$, y que lo consiguen fuera de la universidad (Bautista, 2005).

Los participantes de este estudio perciben, en el $43 \%$, poca propaganda a favor del consumo de tabaco; y el 35,5\%, mucha propaganda. El 43,2 \% declara que no ha observado propaganda en contra de fumar, y el $38,2 \%$ ha visto poca antipropaganda para reducir el consumo de tabaco. Esto indica que las actividades de prevención, promoción y fomento de conductas y estilos de vida saludables aún son muy sutiles y no logran influir en los estudiantes universitarios. Si la publicidad es concebida para nuevos consumidores (Zuzulich et al., 2010), habrá que ejecutar estrategias más intensas y con mayor cobertura para los estudiantes. La práctica de una prevención más activa en los estudiantes universitarios puede hacer el cambio para los grupos de fumadores y no fumadores; en los primeros para prevenir en el futuro nuevos problemas de salud y psicosociales, y para los posibles nuevos formar estilos de vida más saludables.

\section{Referencias}

Antona, M. (2008). "Estudio epidemiológico y educativo en prevención del tabaquismo en estudiantes extemeños/as. Facultad de Educación y Facultad de Formación del profesorado". (Tesis Doctoral). Universidad de Extremadura, España. Recuperado de: http://biblioteca.unex.es/tesis/9788477238461.pdf

Barrueco, M.; Hernández, M., y Torrecilla, M. (2003). Estrategias de prevención, Manual de prevención y tratamiento del tabaquismo. (20. ed.). España: ERGON. Recuperado de: http://www.pnsd.msc.es/Categoria2/ publica/pdf/manual_prevencion_tratamiento_tabaquismo.pdf

Bautista, F. (2005). "Consumo de drogas psicoactivas: lícitas e ilícitas en estudiantes universitarios". Revista Crea Ciencia, (2). Recuperado de: http://scholar.google. es/citations?view_op=view_citation\&hl=es\&user=Ly NOv64AAAAJ\&citation_for_view=LyNOv64AAAAJ:ux608ySG0sC

Calleja, N.; Pick, S.; Reidl, L., y González-Forteza, C. (2010). “Programas de prevención de tabaquismo para mujeres adolescentes". Revista Salud Mental, 33(5), 419-427.

Gómez, A.; Herde, J.; Lafee, A.; Lobo, S., y Martín, E. (2007). "Consumo de drogas lícitas e ilícitas por estudiantes universitarios". Facultad de Ingeniería. Universidad de Carabobo, 2006. Revista de La Facultad de Ciencias de La Salud., 11(3), 41-45.

Kuri-Morales PA., JF, G.-R., JF., Hoy, MJ., \& Cortéz-Ramírez, 
M. (2006). Epidemiología del tabaquismo en México. Salud Pública Mex., 48(1), 591-598.

Poza, C.; Robledo, T.; Fresnillo, L.; Fresnillo, G. y Fernández, C. (s.f.). "Prevención del consumo de alcohol y tabaco. guía didáctica para el profesorado de primer ciclo de ESO". Recuperado de: http://www.msssi.gob. es/profesionales/saludPublica/prevPromocion/docs/ guiaProfesoradoESO.pdf

Prat-Marin, A. (1994). "Epidemiología del tabaquismo en los estudiantes de ciencias de la salud". Revista Saúde Pública. Recuperado de: http://www.scielo.br/pdf/ rsp/v28n2/02.pdf
Sáiz, P.; González, Ma.; Jiménez, L.; Delgado, Y.; Liboreiro, Ma.; Granda, B., y Bobes, J. (1999). "Consumo de alcohol, tabaco y otras drogas y rasgos de personalidad en jóvenes de enseñanza secundaria". Adicciones, 11(3), 209-220.

Sánchez-Zamorano, L. (n.d.). "Adolescencia y tabaquismo". Colección de Documentos de Investigación y Estudios Sobre Juventud.

Zuzulich, M.; Cabieses, B. y Pedrals, N. (2010). "Factores asociados a consumo de tabaco durante el último año en estudiantes de educación superior". Recuperado de: http://www.redalyc.org/articulo. oa? id=105215405009 